\title{
Does the normal four steps weight-bearing rule predict the need for radiography in cases of blunt ankle trauma?
}

\author{
Hassan Amiri ${ }^{1}$, Samad Shams Vahdati ${ }^{2}$, Mehdi Gharehkhani ${ }^{3}$, Radin Maheronnaghsh ${ }^{4}$ \\ Hamid Shokoohi ${ }^{5}$, Vafa Rahimi-Movaghar ${ }^{6 *}$
}

Received: 24 Feb 2016

Published: 16 June 2017

\begin{abstract}
Background: Ankle injuries are one of the most common complaints of patients presenting to emergency departments (ED). The Ottawa ankle rules (OAR) was introduced to help physicians to decide who may require x-ray for blunt injuries. The present study aimed at validating the four steps weight-bearing rule of OAR as a sole criterion.

Methods: This prospective observational study was conducted on 214 patients with acute ankle injury who referred to 3 emergency departments over a 7-months period in 2008. Main outcome measures of this survey included the sensitivity, specificity, positive and negative predictive values, and the likelihood ratios (positive and negative) of the four steps weight-bearing rule.

Results: In this study, 34 fractures were found among the patients. The decision rule had a sensitivity of 0.88 and specificity of 0.61 in detecting all midfoot and ankle fractures. Application of this rule by emergency medicine residents resulted in a $47 \%$ reduction in the use of midfoot and ankle radiography.

Conclusion: Applying a four steps weight-bearing rule as a sole criterion to detect ankle fractures is not as accurate and sensitive as OAR. Solitary application of this rule may lead to an increasing number of missed fractures compared with OAR.
\end{abstract}

Keywords: Ankle injury, Decision rules, Ottawa Ankle Rules, Four Steps Weight Bearing Rule

Copyright@ Iran University of Medical Sciences

Cite this article as: Amiri H, Shams Vahdati S, Gharehkhani M, Maheronnaghsh R, Shokoohi H, Rahimi-Movaghar V. Does the normal four steps weight-bearing rule predict the need for radiography in cases of blunt ankle trauma? Med J Islam Repub Iran. 2017 (16 June);31:32.

https://doi.org/10.14196/mjiri.31.32

\section{Introduction}

Ankle injuries are among the patients' most common complaints in emergency departments (ED). It is estimated that more than $95 \%$ of the patients who refer to the hospital due to ankle or midfoot trauma are x-rayed (1). This policy is safe as it ensures that no fractures are missed, but it entails a high use of resources. Stiell et al. suggested that the Ottawa ankle rules (OAR) might help the physicians to screen patients who do not need ankle $\mathrm{x}$ ray.

The OAR has a set of criteria based on clinical examination (1). It suggests radiographic examination of the ankle under the following conditions: (1) pain in the malleolar zone, (2) inability to bear weight both immediately and in

Corresponding author: Dr Vafa Rahimi-Movaghar, v_rahimi@sina.tums.ac.ir

1. Emergency Medicine Management Research Center, Iran University of Medical Sciences, Tehran, Iran. Tehran, Iran.

2. Instructor of Advanced Trauma Life Support, Local Director of PreHospital Trauma Life Support, Road Traffic Injury Research Center, Tabriz University of Medical Sciences, Tabriz, Iran.

3. Tabriz International hospital, Tabriz, Iran.

4. Tehran University of Medical Sciences, Tehran, Iran.

5. George Washington University Medical Center, Washington, DC, USA.

${ }^{6}$. Sina Trauma and Surgery Research Center, Tehran University of Medical Sciences, Tehran, Iran. the ED (four steps), and (3) bone tenderness at the posterior edge or tip of the lateral or medial malleolus (2-9). They also recommended midfoot radiographs in the following cases: pain in the midfoot $\mathrm{z}$ one, an inability to bear weight both immediately and in the ED (four steps), and bone tenderness at the navicular or the fifth metatarsal (9).

Although interpretation of the bone tenderness item appears to be the most challenging task when applying the rule (2), and while some studies have revealed that even after attending a one-hour training program on the protocol, physicians did not use the OARs (3), other studies have shown that considering only a part of the OAR and

$\uparrow$ What is "already known” in this topic:

Following blunt ankle trauma, radiographic examination of the ankle is suggested if there is pain in the malleolar zone, inability to bear weight both immediately and in the emergency department (four steps), and if there is bone tenderness at the posterior edge or tip of the lateral or medial malleolus.

$\rightarrow$ What this article adds:

Applying a four steps weight-bearing rule as an only principle to detect ankle fractures is not accurate and sensitive. Lonely application of this rule may lead to an increasing number of missed fractures. 
disregarding one of the malleoli will substantially decrease sensitivity (4).

The present study aimed at detecting whether applying weight bearing (four steps test) as a sole criterion is as sensitive as the OAR rule in predicting ankle fracture and identifying the need for ankle and midfoot radiographs.

\section{Methods}

This prospective study was conducted on 214 patients with ankle pain following a blunt trauma, who referred to the emergency departments of Imam Reza and Shohada hospitals in Mian-Do-Ab (Abbasi) in a 7-month period starting September 2008. All patients were clinically examined by an emergency medicine resident (PGY-3) and classified according to their ability to bear weight at the emergency department.

Patients who were younger than 12 or older than 50 years, those with Glasgow Coma Scale (GCS) less than $15 / 15$, those with surgical problems (e.g., abdominal pain or severe chest pain), and those with cardiac diseases with the New York Heart Association (NYHA) Functional Class greater than 2 were excluded from the study.

We used data collection forms to gather the results of physicians' interpretation of radiographs and the four steps test.

All patients were routinely referred for standard ankle radiography series (anteroposterior \& lateral views). The examining physicians interpreted the radiographs at the time of the visit (5). Later, a Board-certified radiologist, who was unaware of physical exam results, interpreted all $\mathrm{X}$-rays.

SPSS software program (Version 16.0, SPSS, Inc., Chicago, IL) was used for statistical analysis. Sensitivity, specificity, positive and negative likelihood ratio, and positive and negative predictive value with a $95 \%$ confi- dence interval were calculated. Chi-square test was used to compare the proportions of patients who were able to bear weight and to yield the negative radiographs between the 2 groups. $\mathrm{P}<0.05$ was considered as statistically significant.

\section{Results}

During the study period, from September 2008 to March 2009, we found that 214 patients were eligible for enrollment, of whom 12 met the exclusion criteria and 2 were missed, leaving a total of $200(93.45 \%)$ patients who were enrolled at 3 centers (Figure 1).

The overall mean $\pm \mathrm{SD}$ age in the study group was $32.58 \pm 8.96$ years, and $147(73.5 \%)$ were male patients. The mechanisms of injury, types of fracture, and their frequencies are demonstrated in Table 1.

The weight bearing (four steps) rule was used to assess all 200 patients; of them, $106(53 \%)$ did not meet the criterion (able to bear weight at least four steps) in the physical examination, of whom 102 had normal radiography results. Also, 94 (47\%) patients met the criterion "unable to bear weight", and the radiography results of 30 patients showed a fracture.

As demonstrated in Table 2, the overall sensitivity and specificity of the four steps test in predicting fractures were $88.2 \%$ (95\% CI:73.4\%-95.3\%) and 61.4\% (95\% CI:53.9\%-68.5\%), respectively. The negative predictive value, negative likelihood ratio, positive predictive value, and positive likelihood ratio were calculated to be 0.96 (95\% CI: 0.90-0.98), 0.19, 0.31 (95\% CI: 0.23-0.41), and 2.28 , respectively $(\mathrm{p}=0.001)$.

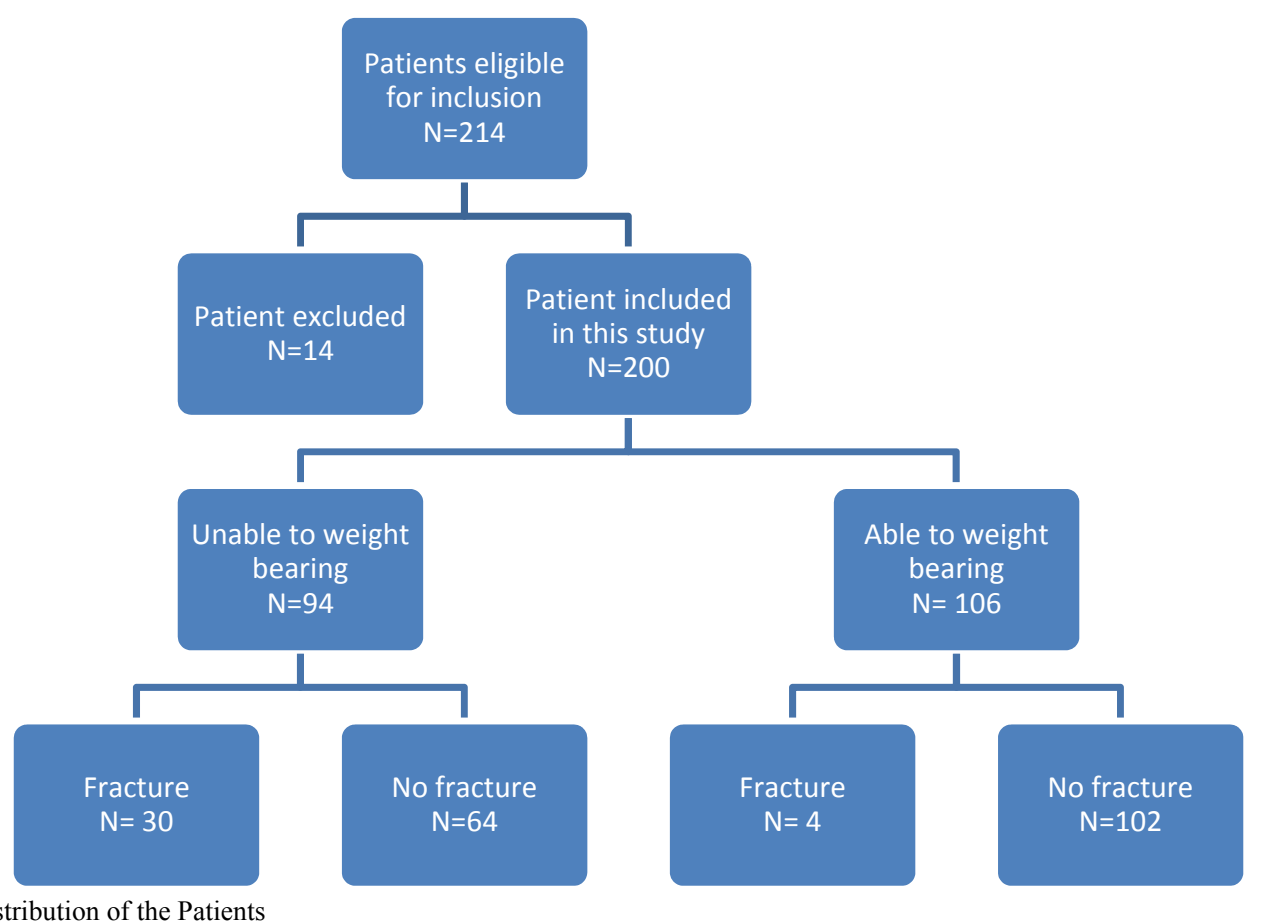

Fig. 1. Distribution of the Patients 
Table 1. Characteristics of the Patients

\begin{tabular}{|c|c|}
\hline Characteristic & Total $(\%)$ \\
\hline \multicolumn{2}{|l|}{ Sex } \\
\hline Male & $147(73.5)$ \\
\hline Female & $53(26.5)$ \\
\hline Age mean $( \pm \mathrm{SD})$, year & $32.58 \pm 8.96$ \\
\hline \multicolumn{2}{|l|}{ Mechanism of injury } \\
\hline Motor vehicle crash & $124(62)$ \\
\hline Direct trauma (ankle sprain) & $64(32)$ \\
\hline Fall & $12(6)$ \\
\hline
\end{tabular}

Table 2. Outcome of Ankle Injuries

\begin{tabular}{lccc}
\hline & \multicolumn{3}{c}{ Radiography } \\
\hline Weight bearing & Fracture & No fracture & Total (\%) \\
Met criterion & 30 & 64 & $94(47)$ \\
Did not meet criterion & 4 & 102 & $106(53)$ \\
\hline
\end{tabular}

\section{Discussion}

This prospective observational study cannot prove that weight bearing in four steps test, as a sole rule, has acceptable sensitivity. We chose only the weight-bearing item of this protocol because we believed that we could decide the ankle radiographies by taking into account only weight-bearing abilities. However, the study did not confirm our hypothesis. Meanwhile, considering the mechanism of injury, motor vehicle accidents consisted of $62 \%$ of our study population. However, in the study of the Ottawa ankle rules (OAR), most patients $(83.9 \%)$ were injured by ankle twisting and only $2 \%$ of the injuries were due to motor vehicle accidents. This major difference in study settings might have affected the results. In addition, most of the patients were male $(73.5 \%)$. Sex differences might have affected the results. Since 1981, several studies have been conducted to establish predictive rules to use radiography in ankle trauma (5-7). Although other studies have shown that the OAR protocol is the most recommended and validated $(2,5,8-10)$, we decided to consider the weight- bearing rule of the protocol separately to confirm the four steps test as a sole criterion.

We found $88.2 \%$ sensitivity in the four steps test. Our results are similar to those of Pigman et al. (11). According to some studies, decision- making based on only one part of OAR is not encouraged $(4,11)$. Moreover, other studies showed that the overall interobserver reliability of ankle physical examination in OAR is acceptable $(1,12)$.

We suggest replicating this study with larger sample sizes in multiple centers and making comparisons with $\mathrm{OAR}$ in the same population. In addition, further suggested evaluations include assessing the weight- bearing validity based on the amount of time elapsed since trauma. Meanwhile, future studies should compare OAR with other rules for their district/ethnicity.

One limitation of this study was its lack of large sample size which included a total of 200 patients who were enrolled into the study from 3 centers during 7 months. Another limitation was failure to completely perform OAR clinical examination to compare the results. The last but not least limitation was related to differences in trauma mechanism between our study and OAR.

\section{Conclusion}

Our prospective study could not prove the validity of weight- bearing (four steps test) as a sole rule to decide whether or not patients with blunt ankle injuries need xray.

\section{Conflict of Interests}

The authors declare that they have no competing interests.

\section{References}

1. Stiell IG, Greenberg GH, McKnight RD, Nair RC, McDowell I, Worthington JR. A study to develop clinical decision rules for the use of radiography in acute ankle injuries. Ann Emerg Med. 1992;21(4):384-90.

2. Can U, Ruckert R, Held U, Buchmann P, Platz A, Bachmann LM. Safety and efficiency of the Ottawa Ankle Rule in a Swiss population with ankle sprains. Swiss Med Wkly. 2008;138(19-20):292-6.

3. Cameron C, Naylor CD. No impact from active dissemination of the Ottawa Ankle Rules: further evidence of the need for local implementation of practice guidelines. CMAJ. 1999;160(8):1165-8.

4. McCann B. Prospective survey to verify the Ottawa ankle rules. J Accid Emerg Med. 2000;17(1):75-6.

5. Stiell IG, Greenberg GH, McKnight RD, Nair RC, McDowell I, Reardon M, et al. Decision rules for the use of radiography in acute ankle injuries. Refinement and prospective validation. JAMA. 1993; 269(9):1127-32.

6. Sujitkumar P, Hadfield JM, Yates DW. Sprain or fracture? An analysis of 2000 ankle injuries. Arch Emerg Med. 1986;3(2):101-6.

7. Kelly AM, Richards D, Kerr L, Grant J, O'Donovan P, Basire K, et al. Failed validation of a clinical decision rule for the use of radiography in acute ankle injury. N Z Med J. 1994;107(982):294-5.

8. Yazdani S, Jahandideh $\mathrm{H}$, Ghofrani $\mathrm{H}$. Validation of the Ottawa Ankle Rules in Iran: a prospective survey. BMC Emerg Med. 2006;6:3.

9. Papacostas E, Malliaropoulos N, Papadopoulos A, Liouliakis C. Validation of Ottawa ankle rules protocol in Greek athletes: study in the emergency departments of a district general hospital and a sports injuries clinic. Br J Sports Med. 2001;35(6):445-7.

10. Auleley GR, Kerboull L, Durieux P, Cosquer M, Courpied JP, Ravaud P. Validation of the Ottawa ankle rules in France: a study in the surgical emergency department of a teaching hospital. Ann Emerg Med. 1998;32(1):14-8.

11. Pigman EC, Klug RK, Sanford S, Jolly BT. Evaluation of the Ottawa clinical decision rules for the use of radiography in acute ankle and midfoot injuries in the emergency department: an independent site assessment. Ann Emerg Med. 1994;24(1):41-5.

12. Stiell IG, McKnight RD, Greenberg GH, Nair RC, McDowell I, Wallace GJ. Interobserver agreement in the examination of acute ankle injury patients. Am J Emerg Med. 1992;10(1):14-7. 